\title{
O Retraimento Social em Adolescentes: Um Estudo Descritivo do seu Ajustamento Sócio-Emocional Segundo a Perspectiva dos Professores
}

\author{
Olívia Ribeiro ${ }^{1}$ \\ António J. Santos \\ Miguel Freitas \\ João V. Correia \\ William James Center for Research, ISPA - Instituto Universitário de Ciências Psicológicas, \\ Sociais e da Vida, Lisboa, Portugal \\ Kenneth Rubin \\ Department of Human Development and Quantitative Methodology, \\ University of Maryland, College Park, MD, United States
}

\begin{abstract}
Resumo
O retraimento social dos adolescentes refere-se ao auto-isolamento relativamente ao seu grupo de pares, que se traduz num comportamento solitário manifestado de forma consistente (em diferentes situações e ao longo do tempo) e na presença de pares com quem têm familiaridade ou não. Este comportamento pode ter consequências negativas, principalmente na adolescência e pode ser um fator preditor de ajustamento psicossocial. Este estudo tem por objetivo analisar a relação entre o retraimento social e o ajustamento sócio-emocional, em termos de comportamento e competências académicas em meio escolar. Participaram no estudo 348 estudantes, com uma média de idades de 14 anos e residência na região da grande Lisboa. Os dados do retraimento social foram recolhidos através da adaptação do Extended Class Play e os do ajustamento sócio-emocional através da adaptação do Teacher-Child Rating Scale. Os resultados mostram que os professores perceberam os adolescentes socialmente retraídos como sendo menos assertivos e como tendo menos aptidões sociais com os pares. Estes resultados apontam para constrangimentos na sua capacidade de ajustamento sócio-emocional à escola.
\end{abstract}

Palavras-chave: Retraimento social, relação de pares, competências sociais, adolescência.

\section{Social Withdrawal in Adolescents: A Descriptive Study of their Socio Emotional Adjustment from the Teacher Perspective}

\begin{abstract}
Adolescents social withdrawal refers to the self-isolation relative to their peer group translating into a solitary behavior manifested consistently (in different situations and over time) and in the presence of either familiar or unfamiliar peers. This behavior can have negative consequences, especially in adolescence and can be a predictor of psychosocial adjustment. This study aims to analyze the relationship
\end{abstract}

Endereço para correspondência: ISPA - Instituto Universitário de Ciências Psicológicas, Sociais e da Vida, Rua Jardim do Tabaco, 34, 1149-041, Lisboa, Portugal. E-mail: asantos@ispa.pt.

Agência de financiamento: Fundação para a Ciência e a Tecnologia - FCT (PTDC/PSI-PDE/098257/2008). 
between social withdrawal and socio-emotional adjustment in terms of behavior and academic skills in the school environment. A total of 348 students participated in the study, with a mean age of 14 years and living in Lisbon metropolitan region. The data of social withdrawal were collected through the Portuguese adaptation of the Extended Class Play and the socio-emotional adjustment data through the Teacher-Child Rating Scale. The results show that teachers perceived the socially withdrawn young as less assertive and as having fewer social skills with peers. These results point to constraints on the ability of social-emotional adjustment to school.

Keywords: Social withdrawal, peer relations, social skills, adolescence.

\section{El Retraimiento Social en Adolescentes: Un Estudio Descriptivo de su Adaptación Socio-Emocional desde la Perspectiva de los Profesores}

\section{Resumen}

El retraimiento social se refiere al auto-aislamiento de los jóvenes en relación con su grupo de pares que se traduce en un comportamiento solitario manifestado constantemente (en diferentes situaciones y con el tiempo) y en presencia, ya sea de los pares familiares o no familiares. Este comportamiento puede tener consecuencias negativas, sobre todo en la adolescencia, y puede ser un factor de pronóstico/ un antecedente de la adaptación psicosocial. El objetivo del presente estudio es analizar la relación entre el aislamiento social y el ajuste socio-emocional en términos de comportamiento y las habilidades en la escuela. Trescientos cuarenta y ocho estudiantes participaron en el estudio, con una edad media de 14 años, y que viven en la región de Lisboa. Los datos del aislamiento social fueron recogidos a través de la adaptación portuguesa del Extended Class Play y los del ajuste socio-emocional a través del Teacher-Child Rating Scale. Los resultados muestran que los profesores perciben a los jóvenes socialmente aislados como menos asertivos y como teniendo menos habilidades sociales con sus pares. Estos resultados apuntan a las limitaciones en la capacidad de ajuste social y emocional a la escuela.

Palabras clave: Aislamiento social, relaciones entre pares, habilidades sociales, adolescencia.

\section{Retraimento Social}

O retraimento social refere-se ao comportamento de crianças e adolescentes que, na presença dos seus pares, se isolam de modo recorrente e consistente, ao longo do tempo e em diferentes contextos (Rubin \& Burgess, 2001; Rubin, Coplan, \& Bowker, 2009). Este constructo está muitas vezes associado à timidez, inibição comportamental, fobia social, reticência social, à passividade e a problemas internalizados (Coplan \& Rubin, 2007; Rubin, Bukowski, \& Parker, 2006; Rubin \& Mills, 1988). No entanto, parece haver consenso entre os investigadores relativamente ao facto de as diferentes formas de retraimento social terem todas em comum a inexistência ou a pouca frequência de interações com os outros (Ladd \& Burgess, 1999). Neste sentido, o retraimento social pode ser entendido como a expressão de diferentes formas de solidão que parecem ter diferentes significados (Rubin \& Mills, 1988), não só, em diferentes momentos do desenvolvimento, como em diferentes contextos sociais (Rubin, Burgess, \& Coplan, 2002), e em diferentes culturas (Chen, Cen, Li, \& He, 2005).

$\mathrm{O}$ termo é utilizado no contexto das relações de pares e refere-se á retirada passiva do sujeito do seu grupo. Neste caso, o défice de interações é o resultado de opções do próprio e pode tomar a designação de retraimento passivo (Rubin \& Coplan, 2004). Os jovens retirados socialmente são aqueles que, na maioria das vezes, preferem estar sós (Oh et al., 2008).

Dada a evidência da importância fundamental das interações sociais, principalmente no período da pré-adolescência e adolescência - em 
que há um aumento significativo do número de membros do grupo de pares e da quantidade de interações sociais - o isolamento social tornou-se num aspeto saliente e preocupante em termos do desenvolvimento e com interesse e relevância para a investigação (Veríssimo \& Santos, 2008). São muitos os estudos que mostram a sua relevância para um desenvolvimento cognitivo, emocional e social adequado. Também é na adolescência que esta questão se reveste de particular importância por ser a etapa do ciclo de vida em que o retraimento social é visto como mais negativo entre os pares e aparecer como forte preditor de problemas futuros a nível social e emocional (Rubin, Wojslawowicz, Burgess, Rose-Krasnor, \& Booth-LaForce, 2006).

Apesar da sua importância, este aspeto foi durante muito tempo negligenciado uma vez que se manifesta através de um comportamento sem nenhuma exuberância. As crianças isoladas socialmente podem ser percebidas como crianças inofensivas, aparentemente calmas, que se entretêm sozinhas e raramente interferem com os outros, sendo que estas características não levantam qualquer preocupação, quer a pais, quer a professores (Rubin \& Burgess, 2001). Estas manifestações de comportamento internalizado são, desde o início da infância, menos salientes e menos prováveis de evocar comportamentos reativos nos outros, ao contrário das manifestações de comportamento externalizado com maior saliência e probabilidade de evocar afetos negativos nos outros (Rubin \& Coplan, 2004). Só cerca dos anos 80 é que se começaram a colocar hipóteses e a investigar as possíveis causas e consequências negativas para o desenvolvimento destas crianças que não interagiam com os seus pares de acordo com a frequência esperada para a sua faixa etária (Rubin, 1982). Os estudos empíricos subsequentes apontam vários tipos de dificuldades, como sejam, socio-emocionais (ansiedade, baixa auto-estima, sintomas depressivos e problemas internalizados), dificuldades com os pares (rejeição, vitimização e qualidade pobre das amizades) e dificuldades escolares (relação professor-aluno de qualidade pobre, dificuldades académicas e evitamento da escola) (Rubin et al., 2009).
Se a capacidade para estabelecer relacionamentos interpessoais positivos e a aceitação dentro do grupo de pares são aspetos centrais no domínio do ajustamento psicossocial é de esperar que crianças e adolescentes retraídos socialmente sejam menos eficazes na concretização de tarefas desenvolvimentais, sociais e académicas comparativamente com crianças que estão mais envolvidas e são mais proactivas socialmente. A investigação revela que, já no pré-escolar, as crianças tímidas/retraídas socialmente raramente iniciam contacto com os seus pares, levam mais tempo a iniciar uma conversa e falam menos frequentemente. Mesmo quando interagem, estas crianças são percebidas pelos seus pares como menos competentes socialmente e como tendo menos sucesso na concretização dos seus objetivos sociais (Crozier \& Perkins, 2002; Rubin, Hymel, \& Mills, 1989). Por outro lado, a literatura sugere que as crianças retiradas socialmente são condescendentes, complacentes com as solicitações dos outros, não assertivas e não directivas quando se aproximam dos seus pares e geralmente bem-comportadas. Com a entrada para a escolaridade, em que quem é dominante parece ser mais popular, estas crianças sossegadas, não assertivas, bem comportadas e até submissas podem não ser vistas como a companhia preferida dos seus pares, ficando à parte do seu grupo (Oh et al., 2008). Para além das dificuldades de relacionamento com os pares, estas crianças ficam ainda sujeitas a dificuldades de aceitação pelo grupo de pares.

\section{Retraimento Social no Contexto da Escola}

No contexto da escola, os comportamentos sócio comunicativos, como sejam a participação verbal e as interações sociais, são considerados contributos importantes para o sucesso da aprendizagem (Daly \& Korinek, 1980). Como referem Coplan, Hughes, Bosacki, e Rose-Krasnor (2011) é, então, de esperar que os professores vejam estes comportamentos de forma positiva e os valorizem. De facto, desde o início da escolaridade, as crianças mais faladoras e mais exuberantes tendem a sentir-se melhor consigo pró- 
prias e são julgadas pelos seus professores como tendo mais competências linguísticas, mais criatividade e como sendo mais inteligentes do que os seus pares menos faladores.

Há estudos que mostram que a quietude das crianças e jovens retraídos socialmente é muitas vezes percecionada pelos professores como uma falta de interesse ou falta de esforço na compreensão das matérias apresentadas nas aulas (Crozier \& Perkins, 2002). Há ainda autores (e.g. Keogh, 2003; Rimm-Kaufman et al., 2002) que referem que são os próprios professores que valorizam estes comportamentos reservados, silenciosos, sossegados e complacentes, dado que ajudam a manter a ordem na sala de aula. O funcionamento da aula depende, em grande parte, do controlo comportamental e emocional dos jovens (Rubin \& Burgess, 2002; Rubin \& Coplan, 2004). Assim, os jovens que manifestam agressividade, impulsividade ou qualquer outra forma de desregulação do comportamento, colocam em risco o funcionamento do grupo, sendo com frequência e precocemente alvo de atenção e acompanhamento mais individualizado. Contrariamente, crianças tipicamente sossegadas, que brincam e trabalham sozinhas e de forma tranquila, representam o modelo de comportamento desejado nestes contextos. Consequentemente, as dificuldades internalizadas, ao contrário das externalizadas, têm maior probabilidade de passarem despercebidas ou serem ignoradas pelos adultos.

Hamre e Pianta (2001) reforçam a ideia de que, para além do funcionamento cognitivo e do comportamento na sala de aula, a capacidade dos jovens para estabelecerem relacionamentos com os outros - e nomeadamente com os seus professores - também prevê o seu posterior ajustamento académico e comportamental à escola. Concomitantemente, as atitudes e crenças dos professores também podem influenciar, direta ou indiretamente, o desenvolvimento social, emocional e académico das crianças. As percepções, atitudes e crenças acerca das características e do comportamento social de cada aluno parecem influenciar a relação de pares e o estabelecimento da relação professor-aluno, bem como, a inferência de traços como sejam a inteligência e as habilidades académicas (Coplan et al., 2011).
Em termos do comportamento de retirada social, alguns estudos põem em evidencia que, tanto os professores como os pares, parecem perceber as crianças retraídas como sendo menos inteligentes (e.g. McCroskey \& Daly, 1976; Richmond, Beatty, \& Dyba, 1985), o que se pode refletir na falta de auto-conceito académico destas crianças que se percecionam a si próprias como menos competentes do ponto de vista escolar e académico (Crozier, 1995). Mesmo, quando têm oportunidade de demonstrar a sua competência académica, as crianças tímidas/retraídas podem evidenciar défices de realização devido ao 'stress' e à ansiedade envolvidos, por exemplo, em situações de avaliação. Os estudos mostram que logo a partir do pré-escolar, estas crianças têm mais predisposição para mostrarem sinais de ansiedade, menos competência social e mais dificuldades académicas, para serem alvo de rejeição pelos pares e para terem uma baixa valorização. Como raramente iniciam trocas com os colegas e respondem menos frequentemente às solicitações dos pares espera-se que tenham poucos amigos dentro do grupo turma.

Também se espera que apresentem o mesmo tipo de comportamentos com os professores, resultando tal circunstância em relações professor-aluno menos próximas (Ladd, \& Burgess, 1999). Requerem mais atenção por parte dos professores (Coplan \& Prakash, 2003) e desenvolvem com eles, uma relação menos próxima e mais dependente uma vez que são menos assertivos e mais submissos do que os seus colegas (Ladd \& Burgess, 1999; Rudasill, Rimm-Kaufman, Justice, \& Pernce, 2006). Hamre e Pianta (2001) referem que crianças cujos relacionamentos com os professores são caracterizados por menos proximidade e maior dependência estão em risco de aumentar a suas dificuldades de ajustamento escolar. Zappulla e Lo Coco (2002), num estudo com crianças e adolescentes em idade escolar, também verificaram que o isolamento social está negativamente correlacionado com a aceitação pelos pares e positivamente associado com uma auto-percepção negativa, assim como, com a identificação de perturbações internalizadas, referida pelos professores. 


\section{Retraimento Social e Sexo}

Nos últimos anos tem-se tornado cada vez mais claro que o sexo é um fator importante nos relacionamentos sociais e desempenha um papel de relevo nas trajetórias desenvolvimentais do retraimento social. Os estudos de Rubin, Bukowski, et al. (2006) mostram que a agressividade é mais aceitável nos rapazes do que nas raparigas e, complementarmente, a timidez é vista como mais aceitável nas raparigas do que nos rapazes. Por outro lado, a timidez parece ser um fator de maior risco e com consequências mais negativas para os rapazes do que para as raparigas (Caspi, Elder, \& Bem, 1988; Morison \& Masten, 1991).

Há evidências que sugerem que, desde a infância até a adolescência, o retraimento social está mais fortemente associado com valores de desajustamento para os rapazes do que para as raparigas (Coplan et al., 2011). Estes resultados foram atribuídos, em parte, á noção de que a timidez é menos aceitável socialmente para os rapazes do que para as raparigas. Neste sentido, é importante realçar o facto de que os rapazes que são socialmente isolados experienciam, a longo prazo, uma maior exclusão, quando comparados com raparigas, dada a menor aceitação deste comportamento nos rapazes.

Também há dados empíricos que mostram que a retirada social se encontra fortemente associada a mais dificuldades sócio emocionais nos rapazes, uma vez que estes acabam por se sentir mais solitários, com pior auto-estima e com maiores probabilidades de serem alvos de rejeição por parte dos pares do que as raparigas (Rubin et al., 2009).

$\mathrm{Na}$ mesma linha de resultados, alguns autores têm apontado para o facto de que é socialmente mais aceitável uma rapariga ser retraída do que um rapaz, principalmente durante a adolescência, onde a conformidade e adesão aos papéis de género se torna especialmente importante. Assim, apesar de ambos os géneros estarem em risco de sofrer de desajustamento emocional e de adversidade entre os pares, o grau relativo deste risco varia de acordo com o período de desenvolvimento (Hinde, Stevenson-Hinde, \& Tamplin, 1985; Rose \& Smith, 2009).
A maioria do que se sabe sobre o fenómeno do retraimento social, seus correlatos, consequências desenvolvimentais e fatores protetores e/ ou de risco, deriva de estudos realizados com amostras norte-americanas, canadianas, asiáticas e centro e norte-europeias. Mesmo nestes contextos têm sido evidenciadas algumas especificidades culturais na avaliação do comportamento de retraimento social. Deste modo, a investigação deste fenómeno em diferentes culturas é, não só importante, como absolutamente necessária (Rubin \& Burgess, 2001). Por outro lado, e apesar do número crescente de estudos sobre esta problemática, a grande maioria ainda se centra sobre os períodos da infância e idade escolar (612 anos), sabendo-se consideravelmente menos sobre as manifestações e implicações da retirada social na adolescência.

Assim, a presente investigação tem como objetivo analisar a relação que existe entre o retraimento social de jovens adolescentes e o ajustamento socio-emocional percebido pelos professores, em termos de comportamentos e competências na escola. Pretende-se analisar o modo como os professores percecionam o ajustamento socio-emocional dos alunos, que são nomeados pelos colegas de turma como retraídos socialmente. Espera-se que os adolescentes retraídos socialmente sejam percebidos como tendo mais dificuldades de ajustamento, quer em termos de comportamento, quer em termos de competências sociais necessárias á adaptação à escola e aos processos de aprendizagem. Finalmente, exploraremos ainda possíveis diferenças de género e idade. Diferentes estudos apontaram para o facto de os rapazes experienciarem maiores dificuldades psicossociais do que as raparigas na infância e idade escolar, mas os resultados são mais contraditórios quando o foco é o período da adolescência (e.g. Gazelle \& Rudolph, 2004).

\section{Método}

\section{Participantes}

Participaram neste estudo 348 estudantes (177 do sexo feminino e 171 do sexo masculino), com idades compreendidas entre os 13 e os 15 anos. Frequentavam os $7^{\circ}$ e $8^{\circ}$ anos de esco- 
laridade em escolas públicas da área da grande Lisboa. Esta amostra faz parte de um projeto de investigação financiado pela FCT (PTDC/PSI-PDE/098257/2008) e aprovado pela comissão de ética. Todos os pais e participantes foram informados do objetivo do estudo e ambos assinaram um consentimento informado antes da recolha de dados.

\section{Instrumentos}

Medida do Retraimento Social. Adaptação portuguesa do Extended Class Play (Correia, Santos, Freitas, Rosado, \& Rubin, 2014; Rubin, Wojslawowicz, et al., 2006). Trata-se de uma medida sociométrica de nomeação pelo grupo de pares adaptada do Revised Class Play (Masten, Morison, \& Pellegrini, 1985). Aplica-se em grupo e pede-se a cada adolescente que se coloque no papel de um realizador de cinema que tem que escolher os protagonistas para o seu próximo filme. Cada adolescente deve nomear um colega do género feminino e um do género masculino para cada um dos 37 papéis que são dados (uns com valência positiva e outros negativa), para que o papel seja o que melhor se adapta ao colega escolhido. Para cada papel não é desejável que se nomeiem a si próprios mas, no caso de isso acontecer, deverá escolher-se adicionalmente um colega do mesmo género. Apenas as nomeações entre sujeitos do mesmo género foram consideradas, evitando possíveis enviesamentos por estereótipos de género (Zeller, Vannatta, Schafer, \& Noll, 2003). Os valores obtidos para os itens foram estandardizados para o género e para a turma, de modo a ajustar o número de nomeações recebidas e o número de nomeadores. A escala tem 37 itens que, através de uma análise factorial, permite avaliar seis dimensões do comportamento social: timidez/ retraimento social $(\alpha=0,85)$, agressividade $(\alpha=$ $0,83)$, exclusão $(\alpha=0,83)$, vitimização $(\alpha=0,86)$, comportamento pró-social $(\alpha=0,74)$ e sociabilidade $(\alpha=0,79)$. Os alfas de Cronbach obtidos para as seis dimensões indicam uma elevada consistência interna.

Medida do Ajustamento Socio-Emocional Percebido pelos Professores. Adaptação portuguesa do Teacher-Child Rating Scale (Hightow- er et al., 1986). Trata-se de um questionário de 38 itens, dirigido a professores, que avalia diferentes aspetos do ajustamento socio-emocional dos jovens, nomeadamente competências na escola e problemas de comportamento. Permite aceder aos constrangimentos e limitações no funcionamento socio-emocional dos jovens. Os itens estão organizados em dois grandes domínios: Problemas de comportamento (18 itens), distribuídos por 3 subescalas (acting-out, timidez/ ansiedade, problemas de aprendizagem), respondidos numa escala de 5 pontos ( $1=$ 'não é um problema' a 5= 'é um problema muito sério'); Competências na escola (20 itens) distribuídas por 4 subescalas (assertividade, orientação para a tarefa, tolerância á frustração, aptidões sociais com os pares), também respondidos numa escala de 5 pontos ( $1=$ 'nada' a $5=$ 'muito').

Os alfas de Cronbach para as 7 subescalas são: acting-out $(\alpha=0,93)$, timidez/ansiedade $(\alpha=$ $0,81)$ e problemas de aprendizagem $(\alpha=0,94)$, assertividade $(\alpha=0,86)$, orientação para a tarefa $(\alpha=0,2)$, tolerância á frustração $(\alpha=0,89) \mathrm{e}$ aptidões sociais com os pares $(\alpha=0,92)$. Globalmente, o domínio dos problemas de comportamento tem um $\alpha=0,93$ e o das competências na escola um $\alpha=0,94$, o que indica uma elevada consistência interna destas medidas.

\section{Procedimento}

Em primeiro lugar foram contactadas as Direcções das Escolas, explicando-se os objectivos e os procedimentos inerentes ao projecto e pediu-se a autorização para proceder à recolha de dados. Com base nos horários escolares das turmas a participar no projecto, elaborou-se uma proposta de recolha de dados que foi entregue aos respectivos Directores de Turma. Também foram entregues aos alunos, pelos Directores de Turma, envelopes dirigidos aos encarregados de educação, que continham os pedidos de consentimento informado para que os seus educandos pudessem participar no projecto e um folheto informativo. Seguidamente, dois investigadores dirigiram-se às várias turmas para explicarem o projecto, quer aos alunos, quer aos professores da disciplina onde as recolhas iriam ocorrer, e esclarecer quaisquer dúvidas. Após a obtenção 
da autorização pelos encarregados de educação e o consentimento dos alunos em participarem no projecto, iniciou-se a recolha de dados.

$\mathrm{O}$ instrumento foi administrado em grupo, em contexto de sala de aula, por dois investigadores. Os alunos foram informados que as suas respostas eram confidenciais, que tinham unicamente fins científicos, que não havia respostas certas ou erradas, e foram instruídos a não discutirem as respostas entre si, uma vez que se pretendia respostas espontâneas e que tivessem a ver com a forma de pensar de cada aluno. Cada sessão durou, aproximadamente, 90 minutos. Finalmente os diretores de turma responderam ao questionário sobre o funcionamento socio-emocional dos jovens.

\section{Análises Estatísticas}

Foram testados os pressupostos de normalidade relativos a cada uma das variáveis em estudo, e dado que se verificou a normalidade ao nível da distribuição utilizaram-se testes paramétricos. Com o intuito de analisar a relação entre as variáveis em estudo foram efetuadas análises de correlações e análises de variância.

\section{Resultados}

\section{Medidas do Retraimento Social e Ajustamento Socio-Emocional em função do Sexo}

Dado que a variável sexo tem uma importância considerável no domínio social, realizou-se uma primeira análise de resultados com o objetivo de verificar se as dimensões do Extended Class Play e os domínios do Teacher-Child Rating Scale variam em função desta variável.

Foi feita uma análise de variância com o teste ANOVA one-way para se analisar a possibilidade de existirem diferenças de resultados, em função do sexo, para as subescalas do Extended Class Play. Nesta análise não foram encontradas diferenças significativas de ao nível da dimensão do retraimento social, $F(1,347)=0,29, p>0,05$, e da dimensão da agressividade, $F(1,347)=0,58$, $p>0,05$.

Foi feita a mesma análise para as subescalas do Teacher-Child Rating Scale. A análise de variância permitiu identificar diferenças significativas de resultados, quer no domínio dos problemas de comportamento, quer no domínio das competências sociais na escola.

Relativamente ao domínio dos problemas de comportamento foram identificadas diferenças na subescala do acting-out, $F(1,347)=4,88$, $p<0,05$. Os resultados mostram que foram os rapazes que obtiveram valores mais elevados $(M=2,38)$ nesta subescala. No domínio das competências sociais na escola foram encontradas diferenças significativas em todas as subescalas. Verificaram-se diferenças significativas na assertividade, $F(1,347)=5,62, p<0,5$, na orientação para a tarefa, $F(1,347)=7,75, p<0,01$, na tolerância á frustração, $F(1,347)=5,44, p<0,05$ e nas aptidões sociais com os pares, $F(1,347)=4,58$, $p<0,05$. Os resultados mostram que as raparigas são mais assertivas $(M=2,98)$, mais orientadas para a tarefa $(M=3,12)$ e mais tolerantes á frustração $(M=2,86)$, enquanto os rapazes são mais aptos socialmente com os pares $(M=3,06)$.

\section{Relação entre o Retraimento Social e o Ajustamento Socio-Emocional Percebido pelos Professores}

Para analisar a relação entre o retraimento social e o ajustamento socio-emocional dos adolescentes, percebido pelos professores, foi feita uma análise correlacional usando o Coeficiente de Correlação de Pearson. Os resultados obtidos mostram que existe uma associação entre o retraimento social e o domínio das competências na escola, nomeadamente na orientação para a tarefa $(r=0,15, p<0,01)$, na assertividade $(r=-$ $0,28, p<0,01)$ e nas aptidões sociais com pares $(r=-0,29, p<0,01)$. Existe uma associação positiva entre o retraimento social e a orientação para a tarefa, e uma associação negativa com a assertividade e as aptidões sociais com os pares. Relativamente aos problemas de comportamento, não se obteve nenhuma correlação significativa para as subescalas deste domínio.

Dado que na análise prévia se encontraram diferenças significativas de resultados em função do sexo, na forma como os professores percecionavam o ajustamento socio-emocional dos adolescentes, procedeu-se a uma nova análise corre- 
lacional (Coeficiente de Correlação de Pearson) distinguindo os rapazes das raparigas. O padrão de correlações manteve-se inalterado para a assertividade e para as aptidões sociais com os pares. No entanto, a orientação para a tarefa só ob-

Tabela 1

Tabela de Resultados do Coeficiente de Correlação entre o Retraimento Social e o Ajustamento Socio-Emocional em Função do Sexo

\begin{tabular}{lcc}
\hline & \multicolumn{2}{c}{ Retraimento Social } \\
\cline { 2 - 3 } Caracterização dos professores & Rapazes & Raparigas \\
\hline Acting-out & $-0,14$ & 0,03 \\
Timidez / Ansiedade & $-0,09$ & 0,08 \\
Problemas de Aprendizagem & $-0,07$ & $-0,03$ \\
Assertividade & $-0,28^{*}$ & $-0,28^{*}$ \\
Orientação para a tarefa & $0,22^{*}$ & 0,10 \\
Tolerância á frustração & 0,16 & $-0,01$ \\
Aptidões Sociais com os Pares & $-0,28^{*}$ & $-0,31^{*}$ \\
\hline
\end{tabular}

${ }^{*} p<0,01$.

\section{Comparação entre o Grupo de Controlo e o Grupo de Adolescentes Socialmente Retraídos}

Tendo em conta o objetivo principal deste estudo procedeu-se à comparação dos resultados do grupo de adolescentes retirados socialmente com os do grupo de controlo. A distribuição dos adolescentes testados pelo grupo de retirados socialmente e pelo grupo de controlo foi feita com base nas nomeações dos pares, através do Extended Class Play, seguindo o procedimento e os critérios já anteriormente utilizados por outros autores (Ladd \& Burgess, 1999; Rubin, Wojslawowicz, et al., 2006). Assim, foram incluídos no Grupo de Retirados os adolescentes cujos valores na dimensão da Timidez/Retraimento Social se encontravam no terço superior (percentil 67) e na dimensão da Agressividade abaixo da mediana e o Grupo de Controlo foi constituído pelos adolescentes que tinham valores abaixo da mediana para estas mesmas dimensões.

Para se verificar se existiam diferenças entre o Grupo de Controlo e o Grupo de Retirados, relativamente à forma como os professores percecionaram o seu ajustamento socio-emocional, teve correlação positiva com o género masculino (ver Tabela 1). Verificou-se que quanto maior é o retraimento social mais os rapazes são orientados para a tarefa. Também não foi encontrada nenhuma associação entre o retraimento social e os problemas de comportamento. 
A análise do Extended Class Play, relativamente á dimensão do retraimento social, não revelou diferenças de resultados em função do sexo contrariamente a outros estudos (Hinde et al., 1985; Rubin, Bukowski, et al., 2006) os quais referem que é socialmente mais aceitável uma rapariga ser retraída do que um rapaz, principalmente na adolescência devido à conformidade e à adesão aos papéis do género. No entanto, as questões das diferenças de sexo evocadas na maioria dos estudos prendem-se com as consequências sociais do comportamento e não com o facto de ele estar mais associado a um género do que a outro.

Quanto à medida do ajustamento socio-emocional dos adolescentes, os resultados variaram em função do género dos sujeitos. No domínio dos problemas de comportamento, constatou-se que no contexto da escola, os rapazes são percebidos como tendo mais comportamentos de acting-out. Este resultado pode ser percebido à luz de resultados de outras investigações que têm mostrado que os relacionamentos interpessoais diferem em função do sexo. Os rapazes tendem a envolver-se em atividades com grupos mais alargados, constituídos por conhecidos, colegas ou amigos, ao contrário das raparigas que tendem a ser mais exclusivas nas suas amizades e a interagirem em díades ou em pequenos grupos de amigas, onde a intimidade é maior (Gifford-Smith \& Brownell, 2003; Poulin \& Chan, 2010). Também Rose e Rudolph (2006) mostram que há diferenças em função do sexo no tipo de relacionamento com os pares em que as raparigas ficam mais fechadas sobre o relacionamento em díade enquanto os rapazes estendem os seus relacionamentos para o grupo. As raparigas parecem ter amizades de maior qualidade, mais intensas e mais íntimas (Parker \& Asher, 1993; Underwood \& Buhrmester, 2007). Assim, pela forma como os rapazes se relacionam, podem ser percebidos em contexto de sala de aula como impulsivos, descontrolados e até mesmo agressivos.

Por outro lado, as perceções, atitudes e crenças acerca do comportamento social de cada aluno também podem conduzir à inferência de traços (Coplan et al., 2011) e há estudos que mostram que os professores têm relações menos conflituosas e mais próximas com as raparigas do que com os rapazes e por outro lado, as raparigas têm mais sucesso académico e registam poucas infracções disciplinares (Hamre \& Pianta, 2001). Esta maior proximidade dos professores e o registo de poucas infrações disciplinares por parte das raparigas pode fazer com que os comportamentos dos rapazes apareçam mais exacerbados no contexto de sala de aula.

Em termos das competências na escola, os resultados mostram que as raparigas são percebidas como sendo mais assertivas, mais orientadas para as tarefas e mais tolerantes à frustração, enquanto os rapazes são percebidos como mais aptos socialmente com os pares. Estes resultados parecem indicar que as raparigas têm melhores indicadores de comportamento de ajustamento á escola. Tal como nos estudos de Hamre e Pianta (2001) as raparigas apresentam hábitos de trabalho significativamente mais positivos do que os rapazes o que é um bom indicador do comportamento de ajustamento á escola. Elas são percebidas como sendo mais confiantes na defesa dos seus pontos de vista, mais atentas, com mais capacidade de se adaptarem aos limites que a escola impõe e com melhores hábitos de trabalho. Estas características parecem indicar um melhor ajustamento à escola. Quanto á melhor competência social do rapazes, ela pode ser explicada pelo que já foi referido anteriormente relativamente às diferenças nos relacionamentos interpessoais entre rapazes e raparigas. As interações para os rapazes baseiam-se na atividade - envolvem desportos e jogos com múltiplos participantes - (Poulin \& Chan, 2010) e estendem-se ao grupo mais alargado.

A análise correlacional entre o retraimento social e o ajustamento socio-emocional percebido pelos professores também revelou diferenças de resultados. O retraimento social está correlacionado positivamente com a orientação para a tarefa e negativamente com a assertividade e com as aptidões sociais com pares. Quer isto dizer que, quanto maior for o retraimento social mais os adolescentes são percebidos como orientados para as tarefas mas são menos capazes de impor e defender pontos de vista, como tendo mais difi- 
culdades em exprimir pensamentos e convicções e como tendo mais dificuldades nas interações e nos relacionamentos com os seus pares (menos competências sociais). Quando considerada a diferença em função do sexo, manteve-se o mesmo padrão de relação mas foram os rapazes que foram percebidos como tendo mais orientados para a tarefa. Se em termos de ajustamento socio-emocional à escola, os rapazes são percebidos como tendo mais comportamentos de acting-out, não é de estranhar que quanto mais retraídos socialmente, mais estejam orientados para a tarefa.

Não se encontrou nenhuma associação entre o retraimento social e os problemas de comportamento. Keogh (2003) e Rimm-Kaufman et al. (2002) referem que são os próprios professores que encorajam e valorizam os comportamentos reservados, silenciosos e complacentes, uma vez que esta é a forma de manter a ordem na sala de aula. Crozier e Perkins (2002) também mostraram que a quietude dos jovens retraídos socialmente é muitas vezes confundida com falta de interesse, pouco esforço, pouca compreensão das matérias apresentadas na aula. Rubin e Coplan (2004) chegam mesmo a afirmar que o comportamento destes jovens representa o modelo de comportamento desejado em contexto escolar.

Finalmente, quando comparados com o grupo de controlo, os adolescentes retraídos socialmente são percebidos pelos professores como sendo menos assertivos e como tendo menos aptidões sociais. Estes resultados traduzem dificuldades de ajustamento à escola. Indicam que os professores percebem estes jovens como tendo menos confiança para defender os seus pontos de vista, menos capacidade de liderar e são menos capazes de enfrentar situações de desafio social sem se retraírem. Mostram, também, que estes jovens têm mais dificuldades de interagir e relacionar-se com outros.

Tal como Rubin et al. (1989) constataram, num estudo com crianças, a timidez/retraimento social leva á perceção de menos competência social e menos sucesso na concretização de objetivos sociais. Se os professores valorizam e avaliam como positivos os comportamentos sociais em sala de aula (Coplan et al., 2011), não é de estranhar que estes adolescentes sejam percebidos como tendo mais dificuldades relacionais quando comparados com os colegas que não são retraídos socialmente.

Estabelecer e manter relacionamentos interpessoais é uma tarefa adaptativa essencial não só na entrada para a escola e na transição de ciclos de escolaridade, mas também para os desafios que emergem em períodos posteriores da vida. As habilidades e competências sociais constituem-se como um indicador de ajustamento social, emocional e cognitivo e também como um fator de risco no desenvolvimento. Em situações mais extremas, os adolescentes retraídos socialmente podem ver aumentadas as suas dificuldades escolares (Rubin et al., 2009), como sejam a qualidade da relação professor-aluno, resultados académicos e até evitamento da escola.

Os resultados deste estudo, relativamente á relação entre o retraimento social e o ajustamento, podem não ser muito expressivos porque não foram considerados sub-tipos de retraimento social. Sub-tipos mais restritos de retraimento social podem ter consequências diferentes e riscos específicos. Por exemplo, os poucos estudos feitos neste domínio mostram que o desinteresse social não está necessariamente associado a problemas sociais e emocionais e parece estar associado a menos ajustamento negativo (Coplan, Prakash, O’Neil, \& Armer, 2004).

A presente investigação é o início de um programa de investigação longitudinal sobre o fenómeno do retraimento social na realidade portuguesa.

$\mathrm{O}$ retraimento social tem sido associado a problemas de internalização (sintomatologia ansiosa e depressiva, solidão ou baixa auto estima), desta forma será importante estudar as trajectórias dos jovens retraídos socialmente de forma a poder prevenir os efeitos desta problemática. Para além das relações com os professores é necessário implementar estudos sobre as relações que estes jovens têm com os seus pais. A literatura tem afirmado que são caracterizadas pela insegurança (e.g. Calkins \& Fox, 1992), por crenças parentais que o filho é vulnerável (e.g. Rubin, Root, \& Bowker, 2010), assim como práticas marcadas pela excessiva 
proteção, intrusividade e autoridade quanto ao que fazer e como fazer. Contudo, para além da maioria destes dados resultar de investigações realizadas na infância, desconhecemos a existência de estudos sobre este tema no nosso contexto cultural, pelo que é uma área de trabalho que deve ser explorada.

Finalmente, pensamos que compreensão do fenómeno do retraimento social deve incidir nas dimensões individual, familiar, social e académica ao longo desenvolvimento. Será fundamental identificar os riscos intra e interpessoais e as trajetórias desenvolvimentais de forma a construir eventuais estratégias de prevenção ou intervenção que sejam adequadas para estes adolescentes.

\section{Referências}

Calkins, S. D., \& Fox, N. A. (1992). The relations among infant temperament, security of attachment, and behavioral inhibition at 24 months. Child Development, 63, 1456-1472.

Caspi, A., Elder, G. H., Jr., \& Bem, D. J. (1988). Moving away from the world: Life course patterns of shy children. Developmental Psychology, 24, 824-831.

Chen, X., Cen, G., Li, D., \& He, Y. (2005). Social functioning and adjustment in Chinese children: The imprint of historical time. Child Development, 76, 182-195. doi:10.1111/j.14678624.2005.00838.x

Coplan, R. J., Hughes, K., Bosacki, S., \& Rose-Krasnor, L. (2011). Is silence golden? Elementary School teachers' strategies and beliefs regarding hypothetical shy/quiet and exuberant/talkative children. Journal of Educational Psychology, 103(4), 939-951. doi:10.1037/a0024551

Coplan, R. J., Prakash, K., O’Neil, K., \& Armer, M. (2004). Do you ' want' to play? Distinguishing between conflicted-shyness and social disinterest in early childhood. Developmental Psychology, 40, 244-258. doi:10.1037/0012-1649.40.2.244

Coplan, R. J., \& Prakash, K. (2003). Spending time with teacher: Characteristics of preschoolers who frequently elicit versus initiate interactions with teachers. Early Childhood Research Quarterly, 18(1), 143-158. doi:10.1016/S08852006(03)00009-7
Coplan, R. J., \& Rubin, K. H. (2007). Social withdrawal in childhood: Conceptual approaches, definitions, and methodological issues. In A. S. Lo Coco, K. H. Rubin, \& C. Zappulla (Eds.), L'isolamento sociale durante l'infanzia [Social withdrawal in childhood] (pp. 1-24). Milan, Italy: Unicopli.

Correia, J., Santos, A. J., Freitas, M., Rosado, A., \& Rubin, K. (2014). Análise fatorial confirmatória do Extended Class Play numa amostra portuguesa de jovens adolescentes. Psicologia: Reflexão e Crítica, 27(3), 462-471. doi:10.1590/16787153.201427306

Crozier, W. R. (1995). Shyness and self-esteem in middle childhood. British Journal of Educational Psychology, 65(1), 85-95. doi:10.1111/j.2044-8279.1995.tb01133.x

Crozier, W. R., \& Perkins, P. (2002). Shyness as a factor when assessing children. Educational Psychology in Practice, 18(3), 239-244. doi:10.1080/0266736022000010267

Daly, J. A., \& Korinek, J. (1980). Interaction in the classroom: An overview. In D. Nimmo (Ed.), Communication Yearbook IV (pp. 515-532). New Brunswick, Canada: Transaction Books.

Gazelle, H., \& Rudolph, K. (2004). Moving toward and away from the world: Social approach and avoidance trajectories in anxious solitary youth. Child Development, 75, 829-849. doi:10.1111/ j.1467-8624.2004.00709.x

Gifford-Smith, M., \& Brownell, C. (2003). Childhood peer relationships: Social acceptance, friendships, and peer networks. Journal of School Psychology, 41(4), 235-285. doi:10.1016/S00224405(03)00048-7

Hamre, B., \& Pianta, R. (2001). Early teacher-child relationships and the trajectory of children's school outcomes through eighth grade. Child Development, 72(2), 625-638. doi:10.1111/14678624.00301

Hightower, A. D., Work, W. C., Cowen, E. L., Lotyczewski, B. S., Spinell, A. P., Guare, J. C., \& Rohrbeck, C. A. (1986). The Teacher-Child Rating Scale: A brief objective measure of elementary children's school problem behaviors and competencies. School Psychology Review, 15(3), 393-409.

Hinde, R. A., Stevenson-Hinde, J., \& Tamplin, A. (1985). Characteristics of 3- to 4-years-olds assessed at home and their interactions in pre- 
school. Developmental Psychology, 21(1), 130140. doi:10.1037/0012-1649.21.1.130

Keogh, B. (2003). Temperament in the classroom: Understanding individual differences. Baltimore, MD: Brookes.

Ladd, G. W., \& Burgess, K. B. (1999). Charting the relationship trajectories of aggressive, withdrawn, and aggressive/withdrawn children during early grade school. Child Development, 70(4), 910-929. doi:10.1111/1467-8624.00066

Masten, A. S., Morison, P., \& Pellegrini, D. S. (1985). A Revised Class Play Method of Peer Assessment. Developmental Psychology, 21(1), 523-533. doi:10.1037/0012-1649.21.3.523

McCroskey, J. C., \& Daly, J. A. (1976). Teacher expectations of the communication apprehensive child in the elementary school. Human Communication Research, 3(1), 67-72.

Morison, P., \& Masten, A. (1991). Peer reputation in middle childhood as a predictor of adaptation in adolescence: A seven-year follow-up. Child Development, 6(5), 991-1007. doi:10.1111/j.1467-8624.1991.tb01585.x

Oh, W., Rubin, K. H., Bowker, J. C., Booth-LaForce, C., Rose-Krasnor, L., \& Laursen, B. (2008). Trajectories of social withdrawal from middle childhood to early adolescence. Journal of Abnormal Child Psychology, 36, 553-566. doi:10.1007/s10802-007-9199-z

Parker, J. G., \& Asher, S. R. (1993). Friendship and friendship quality in middle childhood: Links with peer group acceptance and feelings of loneliness and social dissatisfaction. Developmental Psychology, 29(4), 611-621. doi:10.1037/00121649.29.4.611

Poulin, F., \& Chan, A. (2010). Friendship stability and change in childhood and adolescence. Developmental Review, 30, 257-272. doi:10.1016/j. dr.2009.01.001

Richmond, V. P., Beatty, M. J., \& Dyba, P. (1985). Shyness and popularity: Children's views. Western Journal of Speech Communication, 49(2), 116-125.

Rimm-Kaufman, S., Early, D., Cox, M., Saluja, G., Pianta, R., Bradley, R., \& Payne, C. (2002). Early behavioral attributes and teachers' sensitivity as predictors of competent behavior in the kindergarten classroom. Journal of Applied Developmental Psychology 23, 451-470. doi:10.1016/ S0193-3973(02)00128-4
Rose, A. J., \& Rudolph, K. D. (2006). A review of sex differences in peer relationship processes: Potential trade-offs for the emotional and behavioural development of girls and boys. Psychological Bulletin, 132, 98-131. doi:10.1037/00332909.132.1.98

Rose, A. J., \& Smith, R. (2009). Sex differences in peer relationships. In K. H. Rubin, W. M. Bukowski, \& B. Laursen (Eds.), Handbook of peer interactions, relationships, and groups (pp. 379393). New York: Guilford Press

Rubin, K. (1982). Nonsocial play in preschoolers: Necessarily evil? Child Development, 53, 651657.

Rubin, K., Bukowski, W. M., \& Parker, J. G. (2006). Peer interactions, relationships ad groups. In N. Eisenberg (Ed.), Handbook of child psychology ( $6^{\text {th }}$ ed., Vol. 3, Chap. 10, pp. 571-645). Hoboken, NJ: John Wiley \& Sons.

Rubin, K. H., \& Burgess, K. (2001). Social withdrawal and anxiety. In M. W. Vasey \& M. R. Dadds (Eds.), The developmental psychopathology of anxiety (Chap. 18, pp. 407-434). Oxford, UK: Oxford University Press.

Rubin, K. H., \& Burgess, K. B. (2002). Parents of aggressive and withdrawn children. In M. Bornstein (Ed.), Handbook of parenting ( $2^{\text {nd }}$ ed., Vol. 1, pp. 383-418). Hillsdale, NJ: Lawrence Erlbaum.

Rubin, K. H., Burgess, K. B., \& Coplan, R. J. (2002). Social inhibition and withdrawal in childhood. In P. K. Smith \& C. Hart (Eds.), Handbook of childhood social development (pp. 2-47). London: Blackwell.

Rubin, K., \& Coplan, R. (2004). Paying Attention to and not neglecting social withdrawal and social isolation. Merril-Palmer Quarterly, 50(4), 506534. doi:10.1353/mpq.2004.0036

Rubin, K., Coplan, R., \& Bowker, J. (2009). Social withdrawal in childhood. Annual Review of Psychoogy, 60, 141-171. doi:10.1146/annurev. psych.60.110707.163642

Rubin, K. H., Hymel, S., \& Mills, R. S. L. (1989). Sociability and social withdrawal in childhood: Stability and outcomes. Journal of Personality, 57(2), 237-255.

Rubin, K., \& Mills, R. (1988). The many faces of social isolation in childhood. Journal of Consulting and Clinical Psychology, 56(6), 916-924. 
Rubin, K. H., Root, A. K., \& Bowker, J. (2010). Parents, peers, and social withdrawal in childhood: A relationship perspective. New Directions for Child and Adolescent Development, 127, 79-94. doi:10.1002/cd.264

Rubin, K. H., Wojslawowicz, J. C., Burgess, K. B., Rose-Krasnor, L., \& Booth-LaForce, C. L. (2006). The best friendships of shy/withdrawn children: Prevalence, stability, and relationship quality. Journal of Abnormal Child Psychology, 34, 139-153. doi:10.1007/s10802-005-9017-4

Rudasill, K. M., Rimm-Kaufman, S. E., Justice, L. M., \& Pernce, K. (2006). Temperament and language skills as predictors of teacher-child relationship quality in preschool. Early Education Development, 17, 271-291. doi:10.1207/ s15566935eed1702_4

Underwood, M. K., \& Buhrmester, D. (2007). Friendship features and social exclusion: An observation study examining gender and social context. Merrill-Palmer Quarterly, 53(3), 412-438.
Veríssimo, M., \& Santos, A. J. (2008). Desenvolvimento social: Algumas considerações teóricas. Análise Psicológica, 3(26), 389-394.

Zappulla, C., \& Lo Coco, A. (2002). Adjustment at school: The role of social isolation. Etá Evolutiva, 71(1), 99-105.

Zeller, M., Vannatta, K., Schafer, J., \& Noll, R. B. (2003). Behavioral reputation: A cross-age perspective. Developmental Psychology, 39(1), 129139. doi:10.1037/0012-1649.39.1.129
Recebido: 27/01/2014

$1^{a}$ revisão: $24 / 04 / 2014$ Aceite final: 14/07/2014 